\title{
The Restricted Detour Polynomial of the Theta Graph
}

Herish O. Abdullah herish_omer69@yahoo.com Dept. of Math., College of Science University of Salahaddin/ Erbil

Iraqi Kurdistan Region. Iraq

Received on: 01/12/2013

Ivan D. Ali

ivan_dler@yahoo.com

Dept. of Math., College of Education

University of Salahaddin/ Erbil

Iraqi Kurdistan Region. Iraq

Accepted on: 12/04/2014

\section{ABSTRACT}

The restricted detour distance $D^{*}(u, v)$ between two vertices $\mathrm{u}$ and $\mathrm{v}$ of a connected graph $\mathrm{G}$ is the length of a longest $u-v$ path $P$ in $G$ such that $\langle V(P)\rangle=P$. The main goal of this paper is to obtain the restricted detour polynomial of the theta graph. Moreover, the restricted detour index of the theta graph will also be obtained.

Keywords: Restricted detour distance, restricted detour polynomial, Theta graphs.

متعددة حدود الألتفاف المقيدة للبيان ثيتا

$$
\text { قابفم الرياضيات - كلية التربية دلية علية الدين - أربيل }
$$

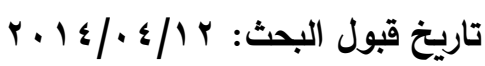

$$
\text { قسم الرياضيات - كلية العلوم عبل الله }
$$

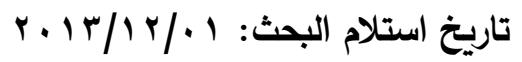

\section{(الملخص}

$$
\begin{aligned}
& \text { تعرف مسافة الألتفاف المقيدة }
\end{aligned}
$$

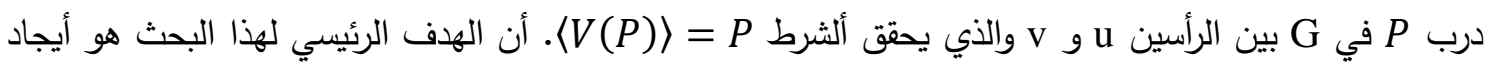

$$
\begin{aligned}
& \text { متعددة حدود الألتفاف المقيد للبيان ثيتا، وكذلك تم الحصول على دلى دليل الألتفاف المقيدة للبيان ثيتا. } \\
& \text { الكلمات المفتاحية: مسافة الالتفاف المقيدة، متعددة حدود الالتفاف المقيدة، بيانات ثيتا. }
\end{aligned}
$$

\section{Introduction}

In this paper, we are concerned only with finite connected simple graphs. We refer the reader to $[1,3,4,5,6]$ for details on graphs, distances in graphs and graph based polynomials. The idea of the restricted detour polynomials was first introduced by Abdullah and Muhammed-Salih[2]. They obtained the restricted detour polynomials and restricted detour indices of some compound graphs.

Let $G$ be a connected graph, the (standard) distance between two vertices $u$ and $v$ of $G$, denoted $d(u, v)$, is the number of edges in a shortest $u-v$ path in $G$. The restricted detour distance $D^{*}(u, v)$ between two vertices $u$ and $v$ of $G$ is the length of a longest $u-v$ path $P$ in $G$ such that $\langle V(P)\rangle=P$. An induced $u-v$ path of length $D^{*}(u, v)$ is called a detour path [4]. The restricted detour polynomial $[2,8]$ of the graph $G$, denoted by $D^{*}(G ; x)$ is defined as follows

$$
D^{*}(G ; x)=\sum_{u, v} x^{D^{*}(u, v)},
$$

where the summation is taken over all unordered pairs $u, v$ of vertices of $G$.Moreover, one easily notice that $D^{*}(G ; x)=\sum_{k \geq 0} C^{*}(G, k) x^{k}$, in which $C^{*}(G, k)$ is the number of unordered pairs of vertices $u, v$ of $G$ such that $D_{G}^{*}(u, v)=k$. 
Let $u$ be any vertex of $G$, and let $C^{*}(u, G ; k)$ be the number of vertices $v$ of $G$ such that $D^{*}(u, v)=k$. Then, the polynomial defined by

$$
D^{*}(u, G ; x)=\sum_{k \geq 0} C^{*}(u, G ; k) x^{k}
$$

is called the restricted detour polynomial of vertex $u$.

It is clear that $D^{*}(G ; x)=\frac{1}{2}\left(\sum_{u \in V(G)} D^{*}(u, G ; x)+p\right)$.

Let $P_{k}$ and $C_{k}$ denote the path and the cycle with $k$ vertices, respectively. The restricted detour polynomials of $P_{k}$ and $C_{k}$ is obtained in [2] and given in the following proposition.

\section{Proposition 1.1}

(1) $D^{*}\left(P_{k} ; x\right)=\sum_{i=0}^{k-1}(k-i) x^{i}$.

(2) $D^{*}\left(C_{k} ; x\right)=\left\{\begin{array}{c}k\left(1+x+\sum_{i=\frac{k+1}{2}}^{k-2} x^{i}\right) \quad \text { if } k \text { is odd, } \\ k\left(1+x+\frac{1}{2} x^{k / 2}+\sum_{i=\frac{k}{2}+1}^{k-2} x^{i}\right) \text { if } k \text { is even. }\end{array}\right.$

\section{The Restricted Detour Polynomial of the Theta Graph}

The theta graph[7] $\theta(l, m, n)$ is the graph consisting of three internally disjoint paths with common endpoints $z$ and $y$ and lengths $l+1, m-1$ and $n-1$ as depicted in Figure 2.1(a). In this paper, we focus our attention on the theta graph $\theta(0, m, n)$ or simply $\theta(m, n)$, as shown in Figure 2.1(b). Without loss of generality, we assume $m \leq$ $n$.

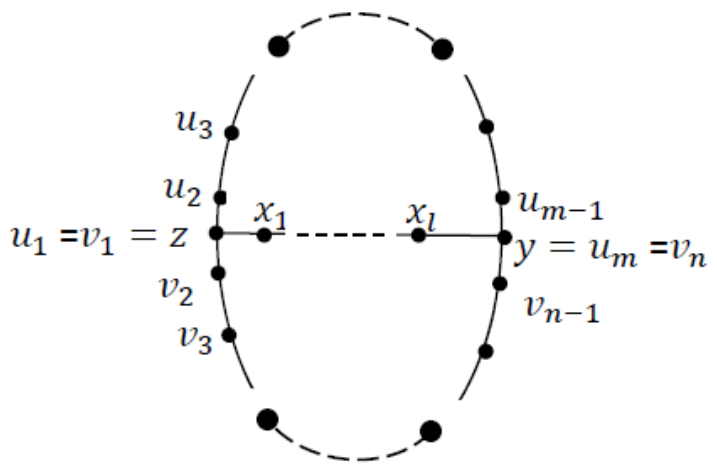

(a) $\theta(l, m, n)$

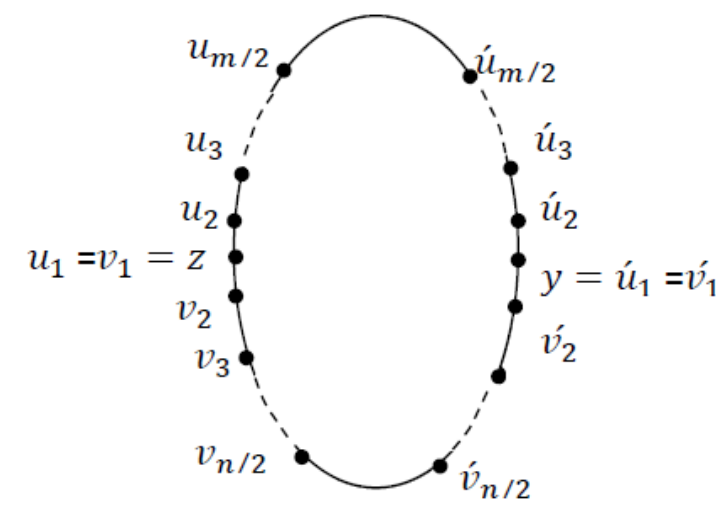

(c) $\theta(m, n), m$ and $n$ are even

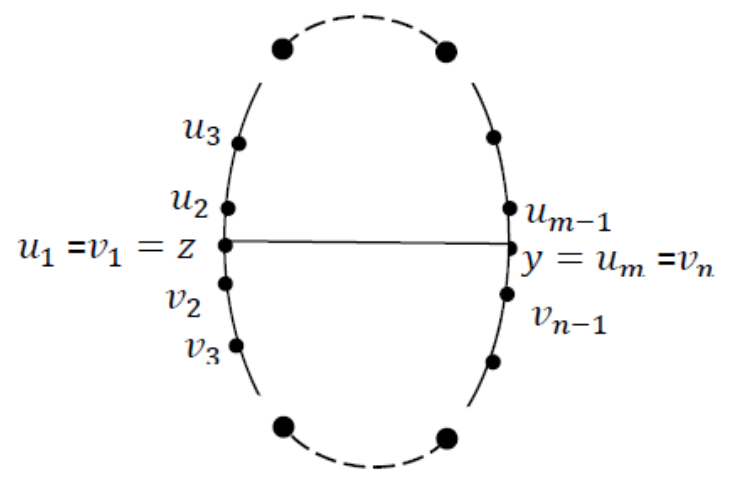

(b) $\theta(m, n)$

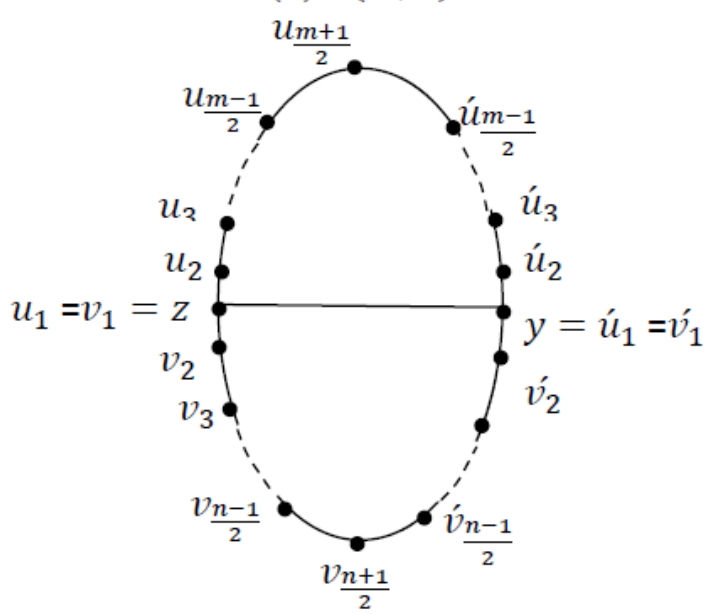

(d) $\theta(m, n), m$ and $n$ are odd

Figure 2.1: The Theta Graph

By simple calculations, we obtain 


$$
\begin{aligned}
& D^{*}(\theta(3,3) ; x)=4+5 x+x^{2}, \\
& D^{*}(\theta(3,4) ; x)=5+6 x+2 x^{2}+2 x^{3}, \\
& D^{*}(\theta(4,4) ; x)=6+7 x+4 x^{2}+2 x^{3}+2 x^{4}, \\
& D^{*}(\theta(4,5) ; x)=7+8 x+2 x^{2}+5 x^{3}+4 x^{4}+2 x^{5}, \\
& D^{*}(\theta(5,5) ; x)=8+9 x+10 x^{3}+2 x^{4}+5 x^{5}+2 x^{6}, \text { and } \\
& D^{*}(\theta(5,6) ; x)=9+10 x+8 x^{3}+6 x^{4}+4 x^{5}+6 x^{6}+2 x^{7} .
\end{aligned}
$$

The restricted detour polynomials of the theta graph $\theta(m, n)$ are obtained in the next results.

Theorem 2.1 For even $m, n \geq 6$, we have

$$
\begin{aligned}
& D^{*}(\theta(m, n) ; x)=D^{*}\left(C_{m} ; x\right)+D^{*}\left(C_{n} ; x\right)-x-2-2 x^{n-1}+2 \sum_{i=2}^{\frac{m}{2}} \sum_{j=2}^{\frac{n}{2}} x^{m+n-(i+j)} \\
& +2 \sum_{i=3}^{\frac{m}{2}} \sum_{j=3}^{\frac{n}{2}} x^{m+n+1-(i+j)}+2 \sum_{j=2}^{\frac{n-m}{2}+2} x^{n-j+1}+2 \sum_{j=\frac{n-m}{2}+3}^{\frac{n}{2}} x^{m-3+j} \\
& +2 \sum_{i=2}^{\frac{m}{2}} x^{n-3+i}
\end{aligned}
$$

in which, $C_{p}$ is a cycle of $p$ vertices.

Proof. Let $u$ and $v$ be any two vertices of $V(\theta(m, n))$. We refer to Figure 2.1(c), and denote

$$
\begin{aligned}
& \quad V_{1}=\left\{u_{2}, u_{3}, \ldots, u_{\frac{m}{2}}\right\}, V_{1}^{\prime}=\left\{u_{2}^{\prime}, u_{3}^{\prime}, \ldots, u_{\frac{m}{2}}^{\prime}\right\}, V_{2}=\left\{v_{2}, v_{3}, \ldots, v_{\frac{n}{2}}\right\} \text { and } V_{2}= \\
& \left\{v_{2}^{\prime}, v_{3}^{\prime}, \ldots, v_{\frac{n}{2}}^{\prime}\right\} .
\end{aligned}
$$

Two main cases can be distinguished for $u$ and $v$

Case I For all possibilities of $u, v \in V_{1} \cup V_{1} \cup\left\{u_{1}, u_{1}^{\prime}\right\}$ (or $u, v \in V_{2} \cup V_{2} \cup\left\{v_{1}, v_{1}\right\}$ ) and notice that the pair $(z, y)$ with $D^{*}(z, y)=1$ and each of the vertices $z$ and $y$ are counted twice, we have the corresponding polynomial $D^{*}\left(C_{m} ; x\right)+D^{*}\left(C_{n} ; x\right)-x-2$.

Case II If $u \in V_{1} \cup V_{1}$ and $v \in V_{2} \cup V_{2}$, then there are four subcases can be distinguished for $u$ and $v$

(1) If $u \in V_{1}$ and $v \in V_{2}$, then it is obvious that the path $P_{1}$,

$P_{1}: u=u_{i}, u_{i+1}, \ldots, u_{\frac{m}{2}}, u_{\frac{m}{2}}^{\prime}, \ldots, u_{2}^{\prime}, u_{1}^{\prime}, v_{2}, \ldots, v_{\frac{n}{2}}, v_{\frac{n}{2}}, \ldots, v_{j}=v$ is a longest $u-v$ path with $\left\langle P_{1}\right\rangle=P_{1}$, for $i=2, \ldots, m / 2$ and $j=2, \ldots, n / 2$.

Evidently, $D^{*}(u, v)=D^{*}\left(u_{i}, v_{j}\right)=m-i+n-j=m+n-(i+j)$.

Similarly, if $u \in \dot{V}_{1}$ and $v \in V_{2}$, we have $D^{*}(u, v)=D^{*}\left(\dot{u}_{i}, \hat{v}_{j}\right)=m+n-(i+j)$, for $i=2, \ldots, m / 2$ and $j=2, \ldots, n / 2$.

Now, for all values of $i$ and $j$, the corresponding polynomial is

$$
F_{1}(x)=2 \sum_{i=2}^{\frac{m}{2}} \sum_{j=2}^{\frac{n}{2}} x^{m+n-(i+j)} .
$$

(2) If $u \in V_{1}-\left\{u_{2}\right\}$ and $v \in V_{2}-\left\{v_{2}\right\}$, then the path $P_{2}$

$P_{2}: u=u_{i}, u_{i+1}, \ldots, u_{\frac{m}{2}}, u_{\frac{m}{2}}^{\prime}, \ldots, u_{2}^{\prime}, u_{1}^{\prime}, u_{1}\left(=v_{1}\right), v_{2}, \ldots, v_{\frac{n}{2}}, v_{\frac{n}{2}}, \ldots, \dot{v}_{j}=v$ is a longest $u-v$ path with $\left\langle P_{2}\right\rangle=P_{2}$, for $i=3, \ldots, m / 2$ and $j=3, \ldots, n / 2$.

In this case, $D^{*}(u, v)=D^{*}\left(u_{i}, v_{j}\right)=m+n-(i+j)+1$.

Similarly, if $u \in V_{1}-\left\{u_{2}^{\prime}\right\}$ and $v \in V_{2}-\left\{v_{2}\right\}$, then

$D^{*}(u, v)=D^{*}\left(u_{\imath}, v_{j}\right)=m+n-(i+j)+1$, for $i=3, \ldots, m / 2$ and $j=3, \ldots, n / 2$.

Now, for all possible values of $i$ and $j$, the corresponding polynomial is 
$F_{2}(x)=2 \sum_{i=3}^{\frac{m}{2}} \sum_{j=3}^{\frac{n}{2}} x^{m+n+1-(i+j)}$.

(3) If $u=u_{2}$ and $v \in V_{2}$; and since $m \leq n$ then there are two subcases can be distinguished

(a) For $j=2, \ldots, \frac{n-m}{2}+2$, the path $P_{3}: u=u_{2}, u_{1}\left(=v_{1}\right), v_{2}, \ldots, v_{\frac{n}{2}}, \dot{v}_{\frac{n}{2}}, \ldots, \dot{v}_{j}=v$ is a longest $u-v$ path with $\left\langle P_{3}\right\rangle=P_{3}$ and has length $n-j+1$. Hence, $D^{*}(u, v)=D^{*}\left(u_{2}, \dot{v}_{j}\right)=n-j+1$.

(b) For $j=\frac{n-m}{2}+3, \ldots, \frac{n}{2}$, the path $\dot{P}_{3}: u=u_{2}, u_{3}, \ldots, u_{\frac{m}{2}}, u_{\frac{m}{2}}^{\prime}, \ldots, u_{2}, u_{1}(=$ $\left.v_{1}^{\prime}\right), v_{2}, \ldots, v_{j}=v$ is a longest $u-v$ path with $\left\langle\dot{P}_{3}\right\rangle=\dot{P}_{3}$, and has length $m-$ $3+j$.

Hence, $D^{*}(u, v)=D^{*}\left(u_{2}, \hat{v}_{j}\right)=m-3+j$.

Similarly, if $u=u_{2}$ and $v \in V_{2}$ then

$D^{*}(u, v)=D^{*}\left(u_{2}, v_{j}\right)=\left\{\begin{array}{l}n-j+1 \text { if } j=2, \ldots, \frac{n-m}{2}+2, \\ m-3+j \text { if } j=\frac{n-m}{2}+3, \ldots, \frac{n}{2} .\end{array}\right.$

Notice that, each of the pairs $\left(u_{2}, v_{2}\right)$ and $\left(u_{2}, v_{2}\right)$ are counted twice with $D^{*}\left(u_{2}, v_{2}\right)=D^{*}\left(u_{2}, v_{2}\right)=n-1$.

Now, for all possible values of $i$ and $j$, the corresponding polynomial is

$$
F_{3}(x)=2 \sum_{j=2}^{\frac{n-m}{2}+2} x^{n-j+1}+2 \sum_{j=\frac{n-m}{2}+3}^{\frac{n}{2}} x^{m-3+j}-2 x^{n-1} .
$$

(4) If $u \in V_{1}$ and $v=v_{2}$ (or $u \in V_{1}$ and $v=v_{2}$ ), then

$D^{*}(u, v)=D^{*}\left(u_{l}, v_{2}\right)=D^{*}\left(u_{i}, v_{2}\right)=n-2+i-1=n-3+i, \quad$ for $\quad i=$ $2, \ldots, m / 2$

This produces the polynomial $F_{4}(x)=2 \sum_{i=2}^{\frac{m}{2}} x^{n-3+i}$.

Adding the polynomials obtained from the cases I and II and simplifying, we get the required result.

Theorem 2.2 For odd $m, n \geq 7$, we have

$$
\begin{aligned}
& D^{*}(\theta(m, n) ; x)=\underset{m-1}{D^{*}}\left(C_{m-1} ; x\right)+D^{*}\left(C_{n} ; x\right) \underset{m-1 n-1}{2} x^{\frac{n-1}{2}+m-2}-x-2-\underset{n-m}{2 x^{n-1}}+x^{\frac{m+n}{2}} \\
& +2 x^{\frac{m-1}{2}+n-2}+2 \sum_{i=2}^{\frac{m-1}{2}} \sum_{j=2}^{\frac{n-1}{2}} x^{m+n-(i+j)}+2 \sum_{i=3}^{\frac{m-1}{2}} \sum_{j=3}^{\frac{n-1}{2}} x^{m+n+1-(i+j)}+2 \sum_{j=2}^{\frac{n-m}{2}+2} x^{n-j+1} \\
& +2 \sum_{j=\frac{n-m}{2}+3}^{\frac{n-1}{2}} x^{m-3+j}+2 \sum_{i=2}^{\frac{m-1}{2}} x^{n-3+i}+2 \sum_{j=3}^{\frac{n-1}{2}} x^{\frac{m-1}{2}+1+n-j}+2 \sum_{i=3}^{\frac{m-1}{2}} x^{\frac{n-1}{2}+1+m-i}
\end{aligned}
$$

Proof. Let $u$ and $v$ be any two vertices of $V(\theta(m, n))$. We refer to Figure 2.1(d), and denote

$$
\begin{aligned}
& V_{1}=\left\{u_{2}, u_{3}, \ldots, u_{\frac{m-1}{2}}\right\}, V_{1}=\left\{u_{2}^{\prime}, u_{3}^{\prime}, \ldots, \dot{u}_{\frac{m-1}{2}}\right\}, V_{2}=\left\{v_{2}, v_{2}, \ldots, v_{\frac{n-1}{2}}\right\} \text { and } V_{2}= \\
& \left\{v_{2}^{\prime}, v_{3}^{\prime}, \ldots, \dot{v}_{\frac{n-1}{2}}\right\}
\end{aligned}
$$

Two main cases can be distinguished for $u$ and $v$ 
Case I For all possibilities of $u, v \in V_{1} \cup V_{1} \cup\left\{u_{1}, u_{1}\right\}$ (or $u, v \in V_{2} \cup V_{2} \cup\left\{v_{1}, v_{1}\right\}$ ) and notice that the pair $(z, y)$ with $D^{*}(x, y)=1$ and each of the vertices are counted twice, we have the corresponding polynomial $D^{*}\left(C_{m} ; x\right)+D^{*}\left(C_{n} ; x\right)-x-2$.

Case II If $u \in V_{1} \cup V_{1}$ and $v \in V_{2} \cup V_{2}$, then there are nine subcases can be distinguished for $u$ and $v$

(1) If $u \in V_{1}$ and $v \in V_{2}$, then it is obvious that the path $P_{1}$,

$P_{1}: u=u_{i}, u_{i+1}, \ldots, u_{\frac{m-1}{2}}, u_{\frac{m+1}{2}}, u_{\frac{m-1}{2}}, \ldots, u_{2}^{\prime}, u_{1}^{\prime}, v_{2}, \ldots, \dot{v}_{\frac{n-1}{2}}, v_{\frac{n+1}{2}}, v_{\frac{n-1}{2}}, \ldots, v_{j}=v$ is a longest $u-v$ path with $\left\langle P_{1}\right\rangle=P_{1}$, for $i=2, \ldots,(m-1) / 2$ and $j=$ $2, \ldots,(n-1) / 2$.

Evidently, $D^{*}(u, v)=D^{*}\left(u_{i}, v_{j}\right)=m-i+n-j=m+n-(i+j)$.

Similarly, if $u \in V_{1}$ and $v \in \hat{V}_{2}$, we have $D^{*}(u, v)=D^{*}\left(\dot{u}_{i}, \dot{v}_{j}\right)=m+n-(i+j)$, for $i=2, \ldots,(m-1) / 2$ and $j=2, \ldots,(n-1) / 2$.

Now, for all such possible values of $i$ and $j$, the corresponding polynomial is

$F_{1}(x)=2 \sum_{i=2}^{\frac{m-1}{2}} \sum_{j=2}^{\frac{n-1}{2}} x^{m+n-(i+j)}$.

(2) If $u \in V_{1}-\left\{u_{2}\right\}$ and $v \in V_{2}-\left\{v_{2}\right\}$, then the path $P_{2}$

$P_{2}: u=u_{i}, u_{i+1}, \ldots, u_{\frac{m-1}{2}}, u_{\frac{m+1}{2}}, u_{\frac{m-1}{2}}, \ldots, u_{2}^{\prime}, u_{1}^{\prime}, u_{1}(=$

$\left.v_{1}\right), v_{2}, \ldots, v_{\frac{n-1}{2}}, v_{\frac{n+1}{2}}, \hat{v}_{\frac{n-1}{2}}, \ldots, \hat{v}_{j}=v$ is a longest $u-v$ path with $\left\langle P_{2}\right\rangle=P_{2}$, for $i=$ $3, \ldots,(m-1) / 2$ and $j=3, \ldots,(n-1) / 2$.

In this case, $D^{*}(u, v)=D^{*}\left(u_{i}, \hat{v}_{j}\right)=m+n-(i+j)+1$.

Similarly, if $u \in V_{1}-\left\{u_{2}^{\prime}\right\}$ and $v \in V_{2}-\left\{v_{2}\right\}$, then

$D^{*}(u, v)=D^{*}\left(\dot{u}_{i}, v_{j}\right)=m+n-(i+j)+1$, for $i=3, \ldots,(m-1) / 2$ and $j=$ $3, \ldots,(n-1) / 2$.

Now, for all such possible values of $i$ and $j$, the corresponding polynomial is $F_{2}(x)=2 \sum_{i=3}^{\frac{m-1}{2}} \sum_{j=3}^{\frac{n-1}{2}} x^{m+n+1-(i+j)}$.

(3) If $=u_{2}$ and $v \in V_{2}$; and since $m \leq n$ then there are two subcases can be distinguished

(a) For $j=2, \ldots, \frac{n-m}{2}+2$, the path

$P_{3}: u=u_{2}, u_{1}=v_{1}, v_{2}, \ldots, v_{\frac{n-1}{2}}, v_{\frac{n+1}{2}}, v_{\frac{n-1}{2}}, \ldots, \dot{v}_{j}=v$ is a longest $u-v$ path with $\left\langle P_{3}\right\rangle=P_{3}$ and has length $n-j+1$.

Hence, $D^{*}(u, v)=D^{*}\left(u_{2}, v_{j}\right)=n-j+1$.

(b) For $j=\frac{n-m}{2}+3, \ldots, \frac{n-1}{2}$, the path

$\dot{P}_{3}: u=u_{2}, u_{3}, \ldots, u_{\frac{m-1}{2}}, u_{\frac{m+1}{2}}, u_{\frac{m-1}{2}}^{\prime}, \ldots, u_{2}^{\prime}, u_{1}^{\prime}\left(=\dot{v}_{1}\right), v_{2}^{\prime}, \ldots, \dot{v}_{j}=v$ is a longest $u-v$ path with $\left\langle\dot{P}_{3}\right\rangle=\dot{P}_{3}$, and has length $m-3+j$. Hence, $D^{*}(u, v)=$ $D^{*}\left(u_{2}, v_{J}\right)=m-3+j$. Similarly, if $u=u_{2}$ and $v \in V_{2}$ then

$D^{*}(u, v)=D^{*}\left(u_{2}, v_{j}\right)=\left\{\begin{array}{l}n-j+1 \text { if } j=2, \ldots, \frac{n-m}{2}+2, \\ m-3+j \text { if } j=\frac{n-m}{2}+3, \ldots, \frac{n-1}{2} .\end{array}\right.$

Notice that, each of the pairs $\left(u_{2}, v_{2}\right)$ and $\left(u_{2}, v_{2}\right)$ are counted twice with $D^{*}\left(u_{2}, v_{2}\right)=D^{*}\left(u_{2}, v_{2}\right)=n-1$.

Now, for all such possible values of $i$ and $j$, the corresponding polynomial is $F_{3}(x)=2 \sum_{j=2}^{\frac{n-m}{2}+2} x^{n-j+1}+2 \sum_{j=\frac{n-m}{2}+3}^{\frac{n-1}{2}} x^{m-3+j}-2 x^{n-1}$. 
(4) If $u \in V_{1}$ and $v=v_{2}$ (or $u \in V_{1}$ and $v=v_{2}$ ), then $D^{*}(u, v)=D^{*}\left(\dot{u}_{i}, v_{2}\right)=D^{*}\left(u_{i}, v_{2}\right)=n-2+i-1=n-3+i, \quad$ for $\quad i=$ $2,3, \ldots,(m-1) / 2$

This produces the polynomial $F_{4}(x)=2 \sum_{i=2}^{\frac{m-1}{2}} x^{n-3+i}$.

(5) If $u=\frac{u_{\frac{m+1}{2}}}{2}$ and $v=\frac{v_{n+1}}{2}$, then,

$D^{*}(u, v)=D^{*}\left(u_{\frac{m+1}{2}}, \frac{v_{n+1}}{2}\right)=\frac{m-1}{2}+1+\frac{n-1}{2}=\frac{m+n}{2}$, and the polynomial is $F_{5}(x)=x^{\frac{m+n}{2}}$.

(6) If $u=\frac{u_{\frac{m+1}{2}}}{2}$ and $v=v_{2}$ (or $u=\frac{u_{\frac{m+1}{2}}}{2}$ and $v=v_{2}$ ), then,

$D^{*}(u, v)=D^{*}\left(u_{\frac{m+1}{2}}, v_{2}\right)=D^{*}\left(u_{\frac{m+1}{2}}, v_{2}\right)=\frac{m-1}{2}+n-2$.

This produces the polynomial $F_{6}(x)=2 x^{\frac{m-1}{2}+n-2}$.

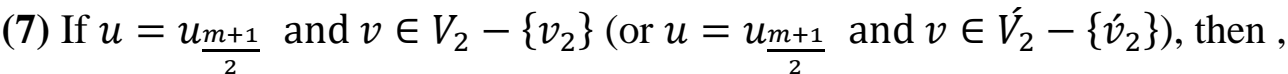
$D^{*}(u, v)=D^{*}\left(u_{\frac{m+1}{2}}, v_{j}\right)=D^{*}\left(u_{\frac{m+1}{2}}, v_{J}\right)=\frac{m-1}{2}+n+1-j$, and the polynomial is $F_{7}(\mathrm{x})=2 \sum_{j=3}^{\frac{n-1}{2}} x^{\frac{m-1}{2}+n+1-j}$.

(8) If $u=u_{2}$ and $v=v_{\frac{n+1}{2}}$ (or $u=u_{2}$ and $v=\frac{v_{n+1}}{2}$ ), then $d^{*}(u, v)=D^{*}\left(u_{2}, \frac{v_{n+1}}{2}\right)=\frac{n-1}{2}+m-2$, and this gives us the polynomial $F_{8}(\mathrm{x})=2 x^{\frac{n-1}{2}+m-2}$.

(9) If $u \in V_{1}-\left\{u_{2}\right\}$ and $v=\frac{v_{n+1}}{2}$ (or $u \in V_{1}-\left\{\dot{u}_{2}\right\}$ and $v=\frac{v_{n+1}}{2}$ ), then $D^{*}(u, v)=D^{*}\left(u_{i}, v_{\frac{n+1}{2}}\right)=D^{*}\left(\dot{u}_{i}, v_{\frac{n+1}{2}}\right)=\frac{n-1}{2}+m+1-i$, and this gives us the polynomial $F_{9}(\mathrm{x})=2 \sum_{i=3}^{\frac{m-1}{2}} x^{\frac{n-1}{2}+m+1-i}$.

Now, adding the polynomials obtained from the Cases I and II and simplifying, we get the required result.

Using the same procedure followed inproving Theorem 2.1 and Theorem 2.2 we obtain the following results.

Theorem 2.3 For odd $m \geq 7$ and even $n \geq 8$, we have

$$
\begin{aligned}
& D^{*}(\theta(m, n) ; x)=D^{*}\left(C_{m} ; x\right)+D^{*}\left(C_{n} ; x\right)-x-2-2 x^{n-1}+2 x^{\frac{m-1}{2}+n-2} \\
& \quad+2 \sum_{i=2}^{\frac{m-1}{2}} \sum_{j=2}^{\frac{n}{2}} x^{m+n-(i+j)}+2 \sum_{i=3}^{\frac{m-1}{2}} \sum_{j=3}^{\frac{n}{2}} x^{m+n+1-(i+j)}+2 \sum_{j=2}^{\frac{n-m-1}{2}+2} x^{n-j+1} \\
& \quad+2 \sum_{j=\frac{n-m-1}{2}+3}^{\frac{n}{2}} x^{m-3+j}+2 \sum_{i=2}^{\frac{m-1}{2}} x^{n-3+i}+2 \sum_{j=3}^{\frac{n}{2}} x^{\frac{m-1}{2}+1+n-j} .
\end{aligned}
$$

Theorem 2.4 For even $m \geq 6$ and odd $n \geq 7$, we have 
$D^{*}(\theta(m, n) ; x)=D^{*}\left(C_{m} ; x\right)+D^{*}\left(C_{n} ; x\right)-x-2-2 x^{n-1}+2 x^{\frac{n-1}{2}+m-2}+$

$2 \sum_{i=2}^{\frac{m}{2}} \sum_{j=2}^{\frac{n-1}{2}} x^{m+n-(i+j)}+2 \sum_{i=3}^{\frac{m}{2}} \sum_{j=3}^{\frac{n-1}{2}} x^{m+n+1-(i+j)}+2 \sum_{j=2}^{\frac{n-1-m}{2}+2} x^{n-j+1}+$

$2 \sum_{j=\frac{n-1-m}{2}+3}^{\frac{n-1}{2}} x^{m-3+j}+2 \sum_{i=2}^{\frac{m}{2}} x^{n-3+i}+2 \sum_{i=3}^{\frac{m}{2}} x^{\frac{n-1}{2}+1+m-i}$

The following results are direct consequences of the Theorems 2.1 and 2.2.

Corollary 2.5 For even $m \geq 6$

$$
\begin{aligned}
& D^{*}(\theta(m, m) ; x)=2 D^{*}\left(C_{m} ; x\right)+2 \sum_{i=2}^{\frac{m}{2}} \sum_{j=2}^{\frac{m}{2}} x^{2 m-(i+j)}+2 \sum_{i=3}^{\frac{m}{2}} \sum_{j=3}^{\frac{m}{2}} x^{2 m+1-(i+j)} \\
& \quad+4 \sum_{j=3}^{\frac{m}{2}} x^{m-3+j}-x+2 x^{m-1}-2 .
\end{aligned}
$$

Corollary 2.6 For odd $m \geq 7$

$$
\begin{gathered}
D^{*}(\theta(m, m) ; x)=2 D^{*}\left(C_{m} ; x\right)+2 \sum_{i=2}^{\frac{m-1}{2}} \sum_{j=2}^{\frac{m-1}{2}} x^{2 m-(i+j)}+2 \sum_{i=3}^{\frac{m-1}{2}} \sum_{j=3}^{\frac{m-1}{2}} x^{2 m+1-(i+j)} \\
\quad+4 \sum_{i=3}^{\frac{m-1}{2}} x^{m-3+i}+4 \sum_{j=3}^{\frac{m-1}{2}} x^{\frac{3 m+1}{2}-j}+4 x^{\frac{3 m-5}{2}}+2 x^{m-1}+x^{m}-x-2 .
\end{gathered}
$$

\section{The Restricted Detour Index of the Theta Graph}

The detour index $d d^{*}(\mathrm{G})$ of a connected graph $G$ is the Wiener index with respect to the restricted detour distance, that is

$$
d d^{*}(G)=\sum_{u, v} D^{*}(u, v)
$$

where the summation is taken over all unordered pairs $u, v$ of vertices of the graph $G$ [2].

It is clear that $d d^{*}(\mathrm{G})=\left.\frac{d}{d x} D^{*}(G ; x)\right|_{x=1}$.

Taking the derivatives of $D^{*}(\theta(m, n) ; x)$ given in the results in Section 2 at $x=$ 1 , we get the restricted detour index of the theta graph $\theta(m, n)$ as is given in the next corollary.

\section{Corollary 3.1}

(1) For even $m, n \geq 6$, we have

$$
d d^{*}(\theta(m, n))=\frac{3}{8}(m+n)\left(m^{2}+n^{2}+m n+\frac{80}{3}\right)-3\left(m^{2}+n^{2}\right)-\frac{11}{2} m n-11 .
$$

(2) For odd $m, n \geq 7$, we have

$$
d d^{*}(\theta(m, n))=\frac{3}{8}(m+n)\left(m^{2}+n^{2}+m n+\frac{83}{3}\right)-3\left(m^{2}+n^{2}\right)-\frac{11}{2} m n-\frac{27}{2}
$$

(3) For odd $m \geq 7$ and even $n \geq 8$, we have

$d d^{*}(\theta(m, n))=\frac{3}{8}\left(m^{3}+n^{3}\right)-3\left(m^{2}+n^{2}\right)+\frac{3}{4}\left(m^{2} n+n^{2} m\right)+\frac{81}{8} m+\frac{41}{4} n-$ $\frac{11}{2} m n-\frac{23}{2}$

(4) For even $m \geq 6$ and odd $n \geq 7$, we have

$$
\begin{aligned}
& d d^{*}(\theta(m, n))=\frac{3}{8}\left(m^{3}+n^{3}\right)-3\left(m^{2}+n^{2}\right)+\frac{3}{4}\left(m^{2} n+n^{2} m\right)+\frac{81}{8} n+\frac{41}{4} m- \\
& \frac{11}{2} m n-\frac{23}{2} .
\end{aligned}
$$

(5) For even $m \geq 6$ we have

$d d^{*}(\theta(m, m))=\frac{9}{4} m^{3}-\frac{23}{2} m^{2}+20 m-11$.

(6) For odd $m \geq 7$, we have

$d d^{*}(\theta(m, m))=\frac{9}{4} m^{3}-\frac{23}{2} m^{2}+\frac{83}{4} m-\frac{27}{2}$.

Proof. Obvious. 


\section{REFERENCES}

[1] Ali, A. A. and Mohammed-Saleh, G. A., (2012); "The Restricted Detour polynomials of a Hexagonal Chain and A Ladder graph", J. Math. Comput. Sci, Vol.2, No. 6, p. 1622-1633.

[2] Abdulla, H. O. and Mohammed-Saleh, G. A., (2010); "Detour Hosoya polynomial of some compound graphs", Rad. J. Comp. Sci. and Maths, Vol.7, No. 1, p. 139154.

[3] Buckly, F. and Harary, F. (1990); "Distance in Graphs", Addison-Wesley, Redwood, California. U. S. A.

[4] Chartrand, G.; Johns, G. L. and Tian S. (1993); "Detour distance in graphs, Annals of Discrete Mathematics", Vol. 55, pp. 127-136.

[5] Gutman, I. (1993); "Some properties of the Wiener polynomial", Graph Theory Notes of New York, Vol.XXV, pp.13-18.

[6] Hosoya, H. (1988); “On some counting polynomials in Chemistry”, Discrete Applied Mathematics, Vol.19, pp.239-257.

[7] Liansheng Tan, Zhongxun Zhu (2010); “The extremal $\theta$-Graphs with respect to Hosoya index and Merrifield-Simmons index", MATCH Commun. Math. Comput. Chem., vol. 63, pp. 789-798.

[8] Mohammed-Saleh, G. A. (2013); "On The Detour Distance and Detour polynomials of graphs", Ph.D. Thesis, University of SalahaddinlErbil, Erbil, Iraq. 